\title{
ON THE ZEROS OF THE ZETA FUNCTION \\ OF THE QUADRATIC FORM $x^{2}+y^{2}+z^{2}$
}

\author{
N. V. PROSKURIN
}

\begin{abstract}
The Epstein zeta function $\zeta_{3}$ of the quadratic form $x^{2}+y^{2}+z^{2}$ is considered. Information is presented about the results of calculating the zeros of $\zeta_{3}$ and of its derivative $\zeta_{3}^{\prime}$. A general setting is suggested for the problem about the distribution of the real parts of the zeros for $L$-functions on the real line.
\end{abstract}

\section{Contents}

Introduction

$\S 1$. On the functions $\zeta_{k}$

$\S 2$. General setting

$\S 3$. Zeros of the function $\zeta_{3}$ and of its derivative

$\S 4$. On functional equations

$\S 5$. On methods of calculation

References

\section{INTRODUCTION}

Let $r_{k}(n)$ be the number of representations of $n$ by the quadratic form $x_{1}^{2}+\cdots+x_{k}^{2}$ over $\mathbb{Z}$, i.e., the number of solutions of the equation $x_{1}^{2}+\cdots+x_{k}^{2}=n$ with $x_{1}, \ldots, x_{k} \in \mathbb{Z}$. For $s \in \mathbb{C}$ with $\operatorname{Re} s>k / 2$, put

$$
\zeta_{k}(s)=\sum_{n=1}^{\infty} \frac{r_{k}(n)}{n^{s}} .
$$

The series on the right converges absolutely and yields an analytic function in the domain $\operatorname{Re} s>k / 2$. The functions $\zeta_{k}$ defined in this way extend analytically to the entire complex plane $\mathbb{C}$ and are known as the Epstein zeta functions of the corresponding quadratic forms (see $\S 1$ below for information on their properties). Our narrowest goal in this paper is to present results concerning the calculation of the zeros of $\zeta_{3}$ and of its derivative. In a broader sense, we shall consider the problem of distribution for the real parts of zeros of the $L$-functions that admit no Euler product expansion. One of such functions is $\zeta_{3}$. Some other functions were considered by the author earlier, see 11, 2]. The distribution of zeros on the complex plane $\mathbb{C}$ and that of their real parts on $\mathbb{R}$ can be characterized by various methods. We adopt the viewpoint presented in $\S 2$. As a result of numerical experiments, in $\S 2$ we formulate a general conjecture about the distribution of zeros of $L$-functions. The results of calculating the zeros of $\zeta_{3}$ and of its derivative are given in the form of histograms in $\S 3$.

The Direchlet series representation $(0.1)$ is good for calculating $\zeta_{3}(s)$ only if the real part of $s$ is sufficiently large. It does not work entirely for calculating $\zeta_{3}(s)$ with $\operatorname{Re} s \leq$ 
$3 / 2$. Quite generally, for the zeta and $L$-functions there are other representations, which, among other things, can be applied to the calculation of values (see $\S \S 4,5)$.

\section{$\S 1$. On THE FUnCTIONS $\zeta_{k}$}

For any $k$, we have the functional equation

$$
\pi^{-s} \Gamma(s) \zeta_{k}(s)=\pi^{-(k / 2-s)} \Gamma(k / 2-s) \zeta_{k}(k / 2-s), \quad s \in \mathbb{C} .
$$

Here and in the sequel, $\Gamma$ is the Euler gamma function. By analogy with the theory of the Riemann zeta function, the line $\operatorname{Re} s=k / 4$ is called the critical line, and the strip $0 \leq \operatorname{Re} s \leq k / 2$ is the critical strip for $\zeta_{k}$. The function $s \mapsto(s-k / 2) \zeta_{k}(s)$ is holomorphic everywhere on $\mathbb{C}$, i.e., the simple pole at the point $k / 2$ is the only singularity of $\zeta_{k}$.

Only few of the functions $\zeta_{k}$ admit Euler product expansions. Such are $\zeta_{k}$ with $k=1,2,4,8$. For them we have

$$
\begin{aligned}
& \zeta_{1}(s)=2 \zeta(2 s) \\
& \zeta_{2}(s)=4 \zeta(s) L(s, \chi), \\
& \zeta_{4}(s)=8\left(1-2^{2-2 s}\right) \zeta(s) \zeta(s-1), \\
& \zeta_{8}(s)=16\left(1-2^{1-s}+2^{4-2 s}\right) \zeta(s) \zeta(s-3), \quad s \in \mathbb{C},
\end{aligned}
$$

where $\zeta$ denotes the Riemann zeta function, $\chi$ is a unique quadratic Direchlet character modulo 4 , and $L(\cdot, \chi)$ is the corresponding Dirichlet $L$-function. The relationship (via the Mellin transformation) between the zeta functions of quadratic forms and the theta functions of quadratic forms is well known. In their turn, the theta functions are modular forms relative to some subgroups of finite index in the group $S L(2, \mathbb{Z})$. For the quadratic forms in $k \geq 4$ variables, application of the theory of modular forms yields formulas that express zeta functions in terms of the Riemann zeta function, the Dirichlet $L$-functions, and $L$-functions of parabolic forms. For example, in the above notation, for $\zeta_{6}$ we have

$$
\zeta_{6}(s)=-4 \zeta(s) L(s-2, \chi)+16 \zeta(s-2) L(s, \chi), \quad s \in \mathbb{C} .
$$

Yet another example is provided by the function

$$
\zeta_{\text {Leech }}(s)=\zeta(s) \zeta(s-11)-L_{\Delta}(s), \quad s \in \mathbb{C},
$$

considered in [2]. Here $L_{\Delta}$ is the $L$-function of the parabolic form $\Delta$ of weight 12 . The coefficients of the Dirichlet series that determines $L_{\Delta}$, i.e., the Fourier coefficients of $\Delta$, are values of the Ramanujan $\tau$-function. The function $s \mapsto \kappa \zeta_{\text {Leech }}(s)$ with $\kappa=$ $65520 / 691$ is the zeta function of the Leech lattice. It can also be treated as the zeta function of a certain quadratic form in 24 variables. The details can be found in Serre's book [3].

There are no formulas like (1.3) and (1.4) for the zeta functions of ternary quadratic forms, and, in particular, for $\zeta_{3}$, because there are no Eisenstein series of weight $3 / 2$, see Hecke [4] and Shimura [5].

The distribution problem for zeros of zeta functions of quadratic forms was treated in various aspects by many authors. As to the functions $\zeta_{k}$ with $k \geq 4$, see Siegel's paper [6]. The important general results were obtained by Ramachandra and Sankaranarayanan [7] (see also [8, 9]).

\section{§2. General Setting}

Here we briefly picture the general viewpoint on the problem of distribution of zeros. Let $L$ be a meromorphic function defined by a Dirickhlet series convergent in some halfplane in $\mathbb{C}$. Let $p$ be a positive real number. All functions tested in our numerical 
experiments satisfy a functional equation of Riemann type that relates $L(s)$ to $L(p-s)$ :

$$
R^{s} \Omega(s) L(s)=R^{p-s} \Omega(p-s) L(p-s),
$$

where $R$ is a positive real number, and $\Omega(s)$ is a product of factors of the form $\Gamma(\alpha s+\beta)$ with some real $\alpha>0$ and $\beta \in \mathbb{C}$. A lot of such functions occur in number theory and the theory of modular forms. In what follows we assume that $L$ satisfies (2.1). The strip $0 \leq \operatorname{Re} s \leq p$ is usually called the critical strip, and the line $\operatorname{Re} s=p / 2$ is the critical line for $L$. The function $L$ may have zeros at poles of the function $\Omega$; there zeros are viewed as trivial, and we exclude them from consideration. Let $N(T)$ be the number of the nontrivial zeros $\rho$ of $L$ with $|\operatorname{Im} \rho|<T$. A typical result looks like this:

$$
N(T)=A T \log T+B T+O(\log T), \quad T \rightarrow \infty,
$$

with some real coefficients $B$ and $A \neq 0$ (a Riemann-Mangoldt type formula).

By the (extended) Riemann hypothesis, the zeros of many $L$-functions admitting the Euler product expansion lie on the critical line. The hypothesis pertains to $\zeta, L(\cdot, \chi)$ (see (1.2)), and to $\zeta_{1}$ and $\zeta_{2}$. Assuming the Riemann hypothesis, from formulas (1.2) we see that $\zeta_{4}$ has zeros on the critical line $\operatorname{Re} s=1$ and on the lines $\operatorname{Re} s=1 / 2$ and $\operatorname{Re} s=3 / 2$, while $\zeta_{8}$ has zeros on the critical line $\operatorname{Re} s=2$ and on the lines $\operatorname{Re} s=1 / 2$ and $\operatorname{Re} s=7 / 2$. For the $L$-functions without Euler product expansion, the distribution of zeros is completely different.

Consider the nontrivial zeros $\rho$ of the function $L$. For an interval $I \subset \mathbb{R}$ and $t \in \mathbb{R}$, put

$$
\nu_{t}(I)=\frac{\sharp\{\rho|\operatorname{Re} \rho \in I,| \operatorname{Im} \rho \mid \leq t\}}{\sharp\{\rho|| \operatorname{Im} \rho \mid \leq t\}} .
$$

This function $\nu_{t}$ is a probability measure, and we can consider the limit $\iota=\lim _{t \rightarrow \infty} \nu_{t}$. To a certain extent, the limit measure $\iota$ (if it exists) characterizes the distribution of the real parts of the zeros of $L$. This simple and natural definition has certain drawbacks. Note that we have $\iota(I)=0$ whenever the numerator in (2.3) is estimated as $o(t \log t)$ as $t \rightarrow \infty$. We can get an additional characteristic it we introduce a certain interval $D \subset \mathbb{R}$, call it the basic interval, put

$$
\nu_{D, t}(I)=\frac{\sharp\{\rho|\operatorname{Re} \rho \in I,| \operatorname{Im} \rho \mid \leq t\}}{\sharp\{\rho|\operatorname{Re} \rho \in D,| \operatorname{Im} \rho \mid \leq t\}},
$$

for each interval $I \subset D$ and $t \in \mathbb{R}$, and then put

$$
\iota=\lim _{t \rightarrow \infty} \nu_{D, t} .
$$

The measure defined in this way depends not only on $L$, but also on the basic interval $D$. In particular, if $D=\mathbb{R}$, then we return to the measure (2.3). If the limit measure exists, then we may raise the question about its density $f$ with respect to Lebesgue measure, i.e., about the representation of the measure $\iota$ in the form

$$
\iota(I)=\int_{I} f(x) d x \quad \text { for each interval } \quad I \subset D .
$$

The measures $\iota$ with appropriately chosen basic intervals $D$ can deliver detailed information on the distribution of zeros. However, we find that in some cases the measure $\iota$ may happen to be almost trivial, but we can get a more interesting measure by modifying the definition somewhat.

We explain the said by an example. Let $D=(c, \infty)$, and let $\psi(t)$ be the denominator on the right-hand side of (2.4). It may happen that $\psi(t) / t \rightarrow \infty$ and

$$
\sharp\{\rho|\operatorname{Re} \rho \in I,| \operatorname{Im} \rho \mid \leq t\}=O(t), \quad t \rightarrow \infty,
$$


for each interval $I=\left(c^{\prime}, c^{\prime \prime}\right)$ with $c^{\prime \prime}>c^{\prime}>c$. Then $\iota(I)=0$ for each such $I$, and $\iota(I)=1$ for each $I=\left(c, c^{\prime \prime}\right)$. We may hope to get a more substantial result if we replace the definition (2.4) by

$$
\nu_{D, t}(I)=\sharp\{\rho|\operatorname{Re} \rho \in I,| \operatorname{Im} \rho \mid \leq t\} \frac{1}{t}
$$

and then define $\iota$ and $f$ as in (2.5) and (2.6). The measures $\nu_{D, t}$ and $\iota$ will no longer be probability ones.

We adopt the following viewpoint:

the problem of describing the distributions of the real parts of the zeros

of $L$-functions is the problem of finding the measures $\iota$ and their densities $f$.

We introduce a general notion. Assume that $L$ is holomorphic everywhere except for finitely many poles. Let $C$ be chosen so that $|\operatorname{Im} \lambda|<C$ for each pole $\lambda$, and put

$$
I(\sigma, T)=\int_{(-T, T) \backslash(-C, C)}|L(\sigma+i t)|^{2} d t, \quad \sigma \in \mathbb{R},
$$

(the interval $(-C, C)$ has been cut off only in order to exclude integration through possible poles). Obviously, for all sufficiently large $\sigma \in \mathbb{R}$ we have $I(\sigma, T)=O(T)$ as $T \rightarrow \infty$. Let $\omega$ be the exact lower bound of the set of such $\sigma$. In Titchmarsh's terminology, this $\omega$ is the abscissa of the half-plane with mean values (see [10]). The two lines given by $\operatorname{Re} s=\omega$ and $\operatorname{Re} s=p-\omega$ will be called the semicritical lines for $L$.

For the functions $\zeta_{k}$ we have $\omega=(k-1) / 2$, and the semicritical lines are $\operatorname{Re} s=$ $(k-1) / 2$ and $\operatorname{Re} s=1 / 2$. In particular, for $\zeta_{3}$ these are $\operatorname{Re} s=1$ and $\operatorname{Re} s=1 / 2$ (see Müller [11 and Fomenko [12, 13]).

Let $N(\sigma, T)$ be the number of zeros $\rho$ of $L$ such that $\operatorname{Re} \rho>\sigma$ and $|\operatorname{Im} \rho|<T$. Under fairly general assumptions on $L$, the following estimate is known:

$$
N(\sigma, T) \ll T, \quad \sigma>\omega \text { and } T \rightarrow \infty .
$$

We may refer to Titchmarsh's book [10]. We see that if the functional equation (2.1) and the asymptotics are valid, then the zeros lie mostly in the strip $p-\omega-\epsilon<\operatorname{Re} s<\omega+\epsilon$.

The calculation of zeros of various $L$-functions has led us to the following observation:

the zeros of L-functions that lie off the critical line are mostly concentrated near the semicritical line.

Moreover, in terms of the densities corresponding to the intervals $D=(p / 2, \omega)$ and $D^{\prime}=(\omega, \infty)$, the following can be observed:

$$
\begin{aligned}
& \text { the density grows monotonically on } D \\
& \text { and decreases monotonically on } D^{\prime} \text {. }
\end{aligned}
$$

We lean on numerical experiments and do not have any proof. A precise statement should pertain to primitive $L$-functions only, and, probably, should include other restrictions. Recall that an $L$-function is primitive of it cannot be represented as the product $L_{1} L_{2}$ of two $L$-functions $L_{1}$ and $L_{2}$ that satisfy the functional equation (2.1) with possibly different $\Omega$ and $R$ but with common $p$.

As an example, consider the function $\zeta_{\text {Leech }}$ defined in (1.4). For it, we have the functional equation (2.1) with $p=12$, the critical line $\operatorname{Re} s=6$, and the semicritical lines $\operatorname{Re} s=23 / 2$ and $\operatorname{Re} s=1 / 2$. Calculations (see [2]) show that the zeros of $\zeta_{\text {Leech }}$ are located on the plane $\mathbb{C}$ in accordance with (2.9) and (2.10). We try to explain this, turning to the expansion (1.4) and adopting the Riemann hypothesis. In the half-plane Re $s \geq 13 / 2+\epsilon$ with $\epsilon>0, \zeta(s)$ differs little from 1 , and the absolute value of $\zeta(s-11)$ is bounded by a constant (depending on $\epsilon$ ). On the contrary, the absolute value of $\zeta(s-11)$ is unbounded on any line $\operatorname{Re} s=q$ with $q \leq 12$. It is natural to expect that the zeros of 
$\zeta_{\text {Leech }}$ are close to those of the function $s \mapsto \zeta(s-11)$ and, consequently, lie near the line $\operatorname{Re} s=23 / 2$.

As yet another example, consider the function $\zeta_{6}$. For it, the line $\operatorname{Re} s=3 / 2$ is critical, and the lines $\operatorname{Re} s=5 / 2$ and $\operatorname{Re} s=1 / 2$ are semicritical. The author's (unpublished) calculations show that the zeros of $\zeta_{6}$ are located on the plane $\mathbb{C}$ in agreement with (2.9) and (2.10). However, we cannot explain this by an argument as above, with (1.3) in place of (1.4).

Also, it is difficult to explain why similar rules are satisfied by the zeros of the function $\zeta_{3}$ and those of the $L$-function associated with the cubic Kubota-Patterson theta function [1]. These functions admit no expansions like (1.3), (1.4).

\section{$\S 3$. Zeros of the Function $\zeta_{3}$ AND of its DeRivative}

First, we note that the nontrivial zeros of $\zeta_{3}$ are located on $\mathbb{C}$ symmetrically relative to the real line $\mathbb{R}$ and the critical line $\operatorname{Re} s=3 / 4$. Therefore, we may restrict ourselves to the zeros that lie in the half-line

$$
\operatorname{Re} s=3 / 4, \quad \operatorname{Im} s \geq 0,
$$

and those lying in the quadrant

$$
\operatorname{Re} s>3 / 4, \quad \operatorname{Im} s \geq 0 .
$$

Our calculation: 1 show that

for $0 \leq \operatorname{Im} s \leq 800000$ there are 493222 zeros

on the half-line (3.1) and 1210949 zeros in the quadrant (3.2);

for $3900000 \leq \operatorname{Im} s \leq 4000000$ there are 63624

zeros on the half-line (3.1) and 191712 zeros in the quadrant (3.2).

The lists of zeros can be found on the site http://www.pdmi.ras.ru/ np. All calculated zeros are simple. The number $N(T)$ of all zeros $\rho$ of the function $\zeta_{3}$ with $0<\operatorname{Im} \rho<T$ has the asymptotics (see [13])

$$
N(T)=\frac{1}{\pi} T \log T-\frac{1}{\pi}(1+\log \pi) T+O(\log T) \quad \text { as } \quad T \rightarrow \infty .
$$

Let $C(T)$ be defined as the number of zeros of $\zeta_{3}$ that lie on the critical line $\operatorname{Re} s=3 / 4$ and satisfy $0<\operatorname{Im} s<T$. We expect that $C(T) \ll T$. From our calculations it can be seen that, as $T$ grows, the ratio $C(T) / T$ stabilizes gradually (near the point 0.6 ).

Now, consider the zeros lying off the critical line. For real $\sigma$ and $T$, we define $N(\sigma, T)$ as the number of zeros $\rho$ for $\zeta_{3}$ that satisfy $\operatorname{Re} \rho>\sigma$ and $0<\operatorname{Im} \rho<T$. We have

$$
\begin{array}{ll}
N(\sigma, T) \ll T, & \text { if } \sigma>1 ; \\
N(\sigma, T) \gg T \log T, & \text { if } \sigma<1 .
\end{array}
$$

Here $T \rightarrow \infty$ and the constants in $\ll$ and $\gg$ depend only on $\sigma$. Estimate (3.4) is a particular case of the main theorem in [7]. this is a fairly deep result. Estimate (3.3) is a consequence of a well-known general principle (see [10, 9.6.2.3]). This is none other than (2.8) applied to $\zeta_{3}$. Estimate (3.3) is also contained in the main theorem in [7].

In order to illustrate the distribution of the real parts of the zeros of $\zeta_{3}$, we compose two histograms (see Figures 1 and 2), showing the zeros calculated. More precisely, the first histogram concerns the zeros $\rho$ with $T-100000 \leq \operatorname{Im} \rho \leq T$, where $T=400000$, and the second concerns the zeros $\rho$ with $0 \leq \operatorname{Im} \rho \leq T$, where $T=800000$. We see a single maximum near the point 1.01 in Figure 1 and a single maximum near the point 1.02 in Figure 2.

\footnotetext{
${ }^{1}$ Earlier, in [14, the zeros were calculated for $0 \leq \operatorname{Im} s \leq 160000$.
} 


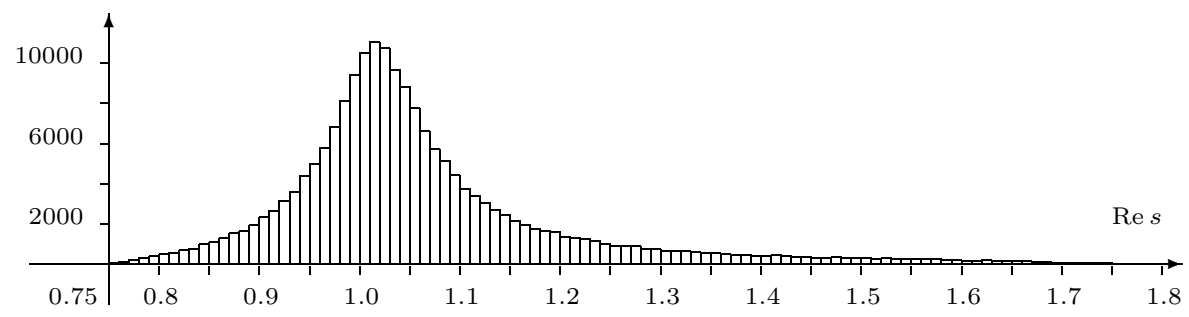

Figure 1. The real axis is split into segments of length 0.01 . If $[a, b]$ is one of them, the height of the column over it is equal to the number of zeros $\rho$ of $\zeta_{3}$ such that $a<\operatorname{Re} \rho \leq b, 3900000<\operatorname{Im} \rho<4000000$.

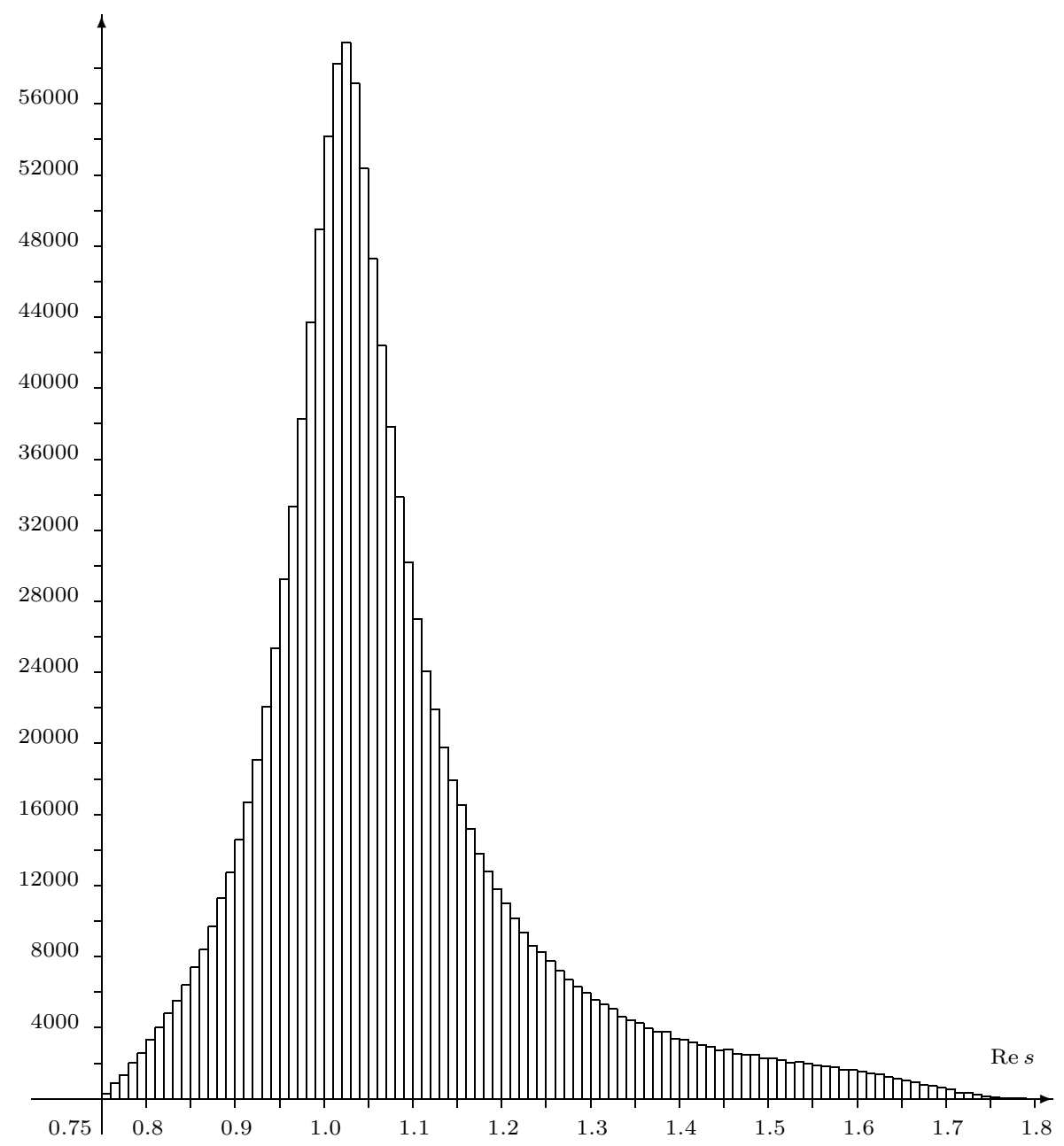

FiguRE 2. The real axis is split into segments of length 0.01 . If $[a, b]$ is one of them, the height of the column over if is equal to the number of the zeros $\rho$ of $\zeta_{3}$ such that $a<\operatorname{Re} \rho \leq b, 0<\operatorname{Im} \rho<800000$.

In histograms of this kind, the maximum point should move to the left, and, in the limit as $T \rightarrow \infty$, will be not greater than 1: this follows from estimates (3.3) and (3.4). The line $\operatorname{Re} s=1$ is semicritical for the function $\zeta_{3}$. We expect (2.9) to hold, so that in the limit as $T \rightarrow \infty$ the maximum point moves to 1 . From the viewpoint adopted 


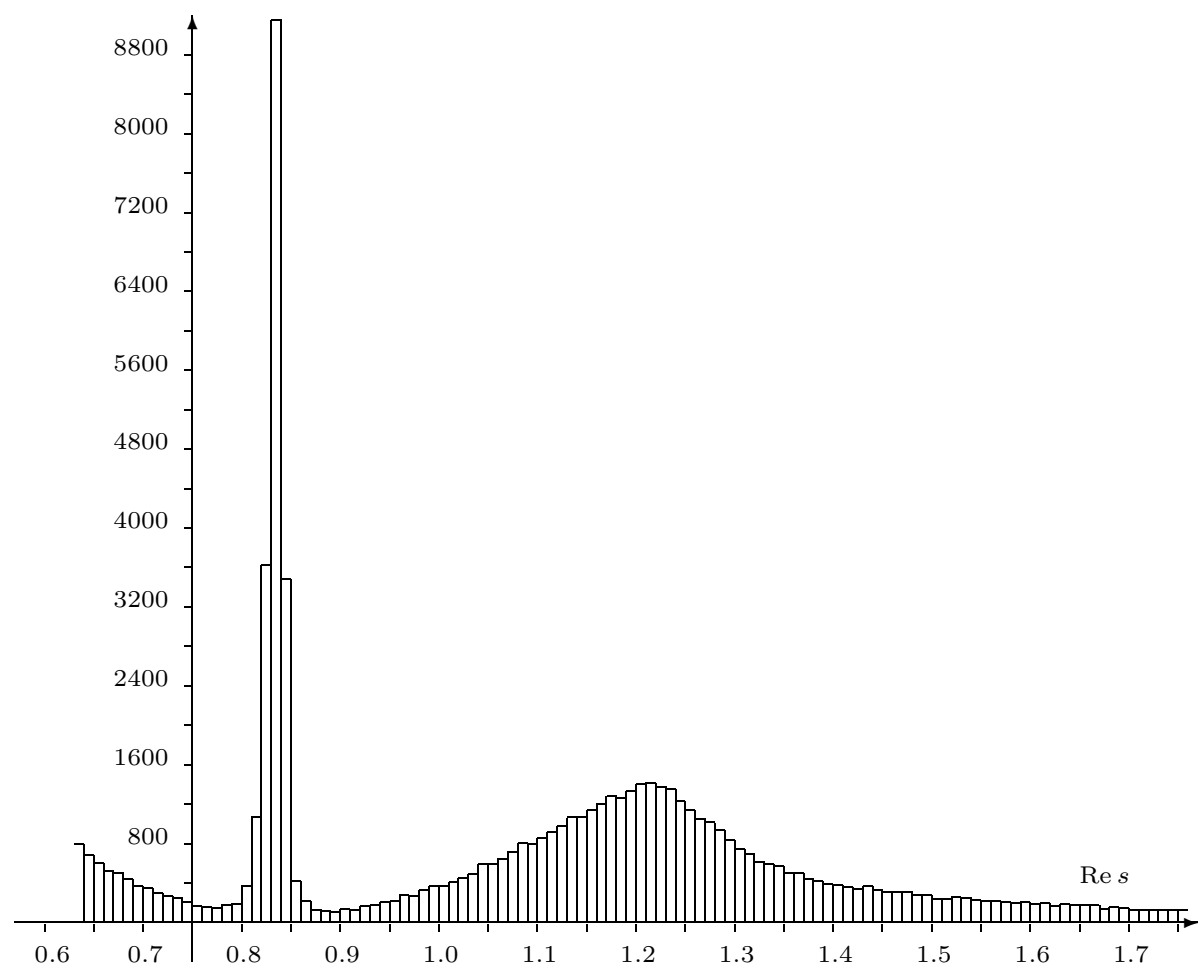

FiguRE 3. The real axis is split into segments of length 0.01 . If $[a, b]$ is one of them, the height of the column over it is equal to the number of the zeros $\rho$ of $\zeta_{3}^{\prime}$ such that $a<\operatorname{Re} \rho \leq b, 768000<\operatorname{Im} \rho<800000$.

in $\S 2$, it is natural to consider the measures $\iota$ and their densities $f$ that correspond to the intervals $D=(3 / 4,1)$ and $D=(1, \infty)$. We expect that not only $(2.9)$ but also the stronger claim $(2.10)$ is true, i.e.,

$$
\begin{aligned}
& \text { the density is monotone increasing on } D=(3 / 4,1) \\
& \text { and is monotone decreasing on } D=(1, \infty) \text {. }
\end{aligned}
$$

The densities $f$ are unbounded, and their behavior near 1 is hard to determine by calculations only. For $D=(1, \infty)$, we mean the density $f$ defined by $(2.5),(2.6)$, and (2.7). It is not clear which of two definitions (2.4) and (2.7) should be taken for $D=(3 / 4,1)$. Choosing the version $(2.4)$ for $D=(3 / 4,1)$, we can observe that the function $x \mapsto a(x-3 / 4)(-\log (1-x)+b)$ with $a$ and $b$ chosen appropriately is a good approximation of the density determined by our calculations. However, we cannot be sure that the density $f$ has this form indeed.

Now we consider the derivative $\zeta_{3}^{\prime}$ of the function $\zeta_{3}$. It has a Direchlet series representation, but admits no functional equation like (2.1). The zeros of $\zeta_{3}^{\prime}$ are symmetric relative to the real line $\mathbb{R}$, but no symmetry occurs relative either to the point $3 / 4$, or to the critical line $\operatorname{Re} s=3 / 4$. Our calculations are presented in two histograms, Figures 3 and 4 . These histograms show that the distribution of the real parts of the zeros of $\zeta_{3}^{\prime}$ has a local maximum near some point $m \approx 0.83$ and the second local maximum near $n \approx 1.2$. The concentration of zeros near the line $\operatorname{Re} s=m$ is extremely high. At first glance, it seems that some function with zeros on the line $\operatorname{Re} s=m$ may be related to $\zeta_{3}^{\prime}$.

However, the situation is not as simple. A thorough look discovers that the maximum point $m$ in Figure 4 is shifted (by about 0.01 ) to the left from the maximum point in Figure 3. The point $n$ of the second maximum is shifted similarly. We also have several other histograms, similar to those in Figures 3 and 4, constructed for the zeros 


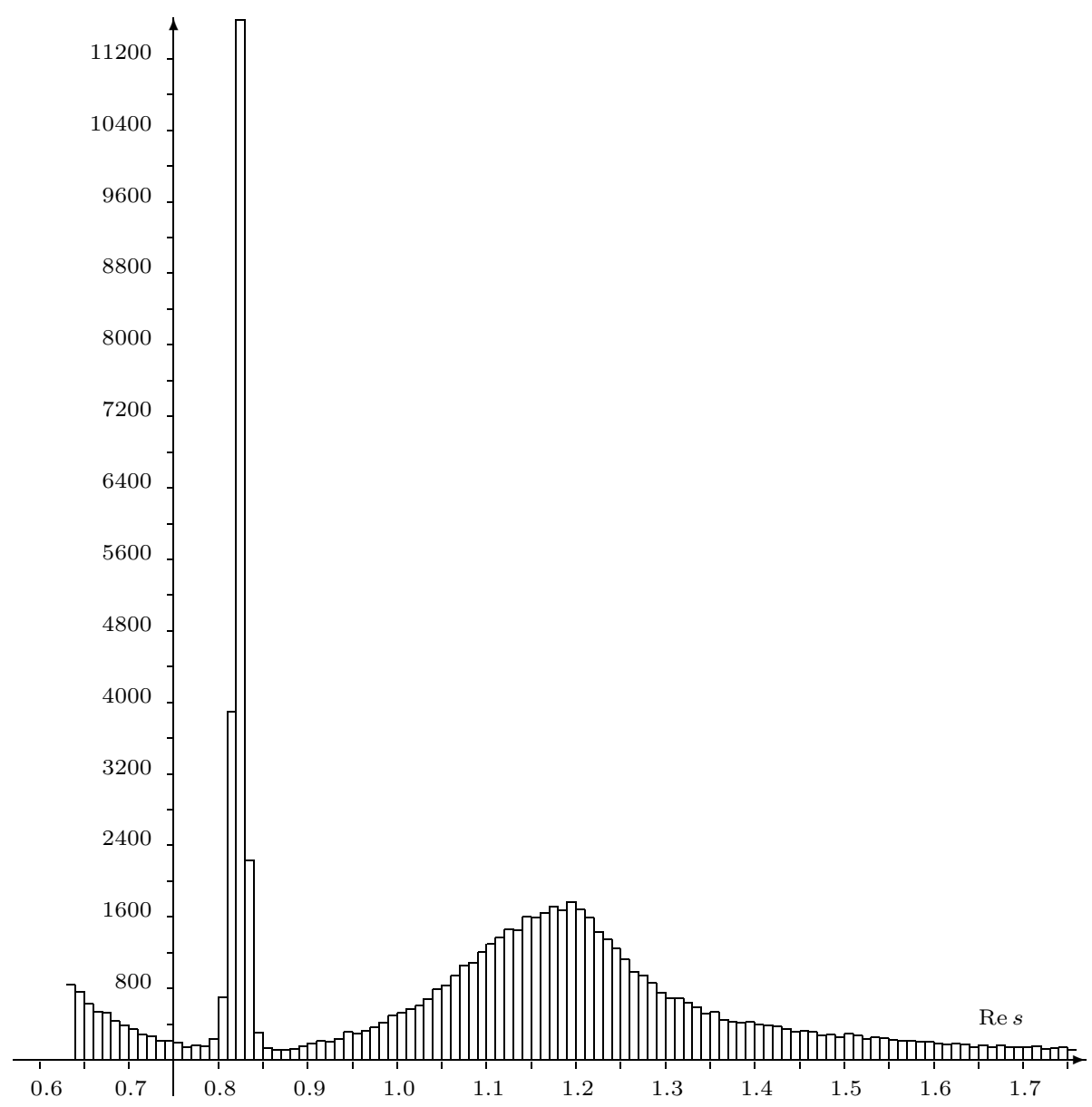

FiguRE 4. The real axis is split into segments of length 0.01 . If $[a, b]$ is one of them, the height of the column over it is equal to the number of the zeros $\rho$ of $\zeta_{3}^{\prime}$ such that $a<\operatorname{Re} \rho \leq b, 3968000<\operatorname{Im} \rho<4000000$.

$\rho$ with $T-32000<\operatorname{Im} \rho<T$ with various $T$. We observe that, when $T$ grows, the corresponding maximum point $m$ moves to the left. At the very beginning, on the histogram built for the zeros $\rho$ with $0<\operatorname{Im} \rho<6000$ we have $m \approx 0.91$. It would be important to understand what are the limit values of $m$ and $n$ as $T \rightarrow \infty$ and to interpret them somehow.

\section{$\S 4$. ON FUNCTIONAL EQUATIONS}

Instead of the series (0.1), the following representation 2 was used for calculating the values of the function $\zeta_{3}$ :

\footnotetext{
${ }^{2}$ Here and in the sequel, $\Gamma$ denotes either the gamma function, or the incomplete gamma function
}

$$
\Gamma(s, z)=\int_{z}^{\infty} \exp (-x) x^{s-1} d x .
$$




$$
\begin{aligned}
\zeta_{3}(s)=\left(\frac{\eta^{s-3 / 2}}{s-3 / 2}-\frac{\eta^{s}}{s}\right) \frac{\pi^{s}}{\Gamma(s)}+\sum_{m=1}^{\infty} \frac{r_{3}(m)}{m^{s}} \frac{\Gamma(s, \pi m \eta)}{\Gamma(s)} \\
+\pi^{2 s-3 / 2} \frac{\Gamma(3 / 2-s)}{\Gamma(s)} \sum_{m=1}^{\infty} \frac{r_{3}(m)}{m^{3 / 2-s}} \frac{\Gamma\left(3 / 2-s, \pi m \eta^{-1}\right)}{\Gamma(3 / 2-s)} .
\end{aligned}
$$

It is valid for all $s \in \mathbb{C}$. As to the parameter $\eta \in \mathbb{C}$, it is only assumed that $\operatorname{Re} \eta>0$. Such representations with a free parameter $\eta$ can be written for other zeta and $L$-functions. For example, for the Riemann zeta function we have

$$
\begin{aligned}
\zeta(s)=\left(\frac{\eta^{s / 2-1 / 2}}{s-1}-\frac{\eta^{s / 2}}{s}\right) \frac{\pi^{s / 2}}{\Gamma(s / 2)}+\sum_{m=1}^{\infty} \frac{1}{m^{s}} \frac{\Gamma\left(s / 2, \pi m^{2} \eta\right)}{\Gamma(s / 2)} \\
+\pi^{s-1 / 2} \frac{\Gamma(1 / 2-s / 2)}{\Gamma(s / 2)} \sum_{m=1}^{\infty} \frac{1}{m^{1-s}} \frac{\Gamma\left(1 / 2-s / 2, \pi m^{2} \eta^{-1}\right)}{\Gamma(1 / 2-s / 2)}
\end{aligned}
$$

The representations like (4.1), (4.2) will be called functional equations with free parameters. Some other names are also used: "approximate functional equation", "shortened functional equation" (as well as the English-language term "smoothed approximate functional equation"). In the most general context, the functional equations with free parameters were deduced by Lavrik (see [15, 16]). Some particular cases of $L$-functions and parameters $\eta$ were considered earlier by many authors; this was not related to the problem of calculating the values of $L$-functions. An ever principal problem is that of approximation of $L$-functions by short Dirichlet series. Such an approximation can be obtained by choosing the parameter $\eta$ appropriately. In the examples of $L$-functions treated here, we can choose the parameter 3 so that the ratios $\Gamma(s, \pi m \eta) / \Gamma(s)$ in $(4.1)$ and $\Gamma\left(s / 2, \pi m^{2} \eta\right) / \Gamma(s / 2)$ in (4.2) differ little from 1 for all $m$ from 1 to some bound, and then decay rapidly in absolute value and tend to 0 as $m \rightarrow \infty$. We state the results that can be obtained on this way.

Let $s \in \mathbb{C}$, and let $a, b, c, d, x, y$ be positive real numbers such that

$$
|\operatorname{Re} s| \leq a, \quad|\operatorname{Im} s| \geq b, \quad c \leq y / x \leq d .
$$

If $2 \pi x y=|\operatorname{Im} s|$, then we have

$$
\zeta(s)=\sum_{m \leq x} \frac{1}{m^{s}}+\pi^{s-1 / 2} \frac{\Gamma(1 / 2-s / 2)}{\Gamma(s / 2)} \sum_{m \leq y} \frac{1}{m^{1-s}}+O\left(T^{-\operatorname{Re} s / 2}\right),
$$

and if $\pi^{2} x y=|\operatorname{Im} s|^{2}$, then

$$
\zeta_{3}(s)=\sum_{m \leq x} \frac{r_{3}(m)}{m^{s}}+\pi^{2 s-3 / 2} \frac{\Gamma(3 / 2-s)}{\Gamma(s)} \sum_{m \leq y} \frac{r_{3}(m)}{m^{3 / 2-s}}+O\left(T^{(1-\operatorname{Re} s) / 2} \log T\right) .
$$

Here $T=|\operatorname{Im} s|$, and the constants in $O$ depend on $a, b, c, d$ only.

Observe that formula (4.2) with $\eta=1$ immediately implies the invariance of the function $s \mapsto \pi^{-s / 2} \Gamma(s / 2) \zeta(s)$ under the change $s \mapsto 1-s$, i.e., the Riemann functional equation

$$
\pi^{-s / 2} \Gamma(s / 2) \zeta(s)=\pi^{-1 / 2+s / 2} \Gamma(1 / 2-s / 2) \zeta(1-s) .
$$

Conversely, the functional equation (4.2) can be deduced from the Riemann equation (4.3) by considering the integral

$$
\int_{\operatorname{Re} z=\epsilon} \pi^{-z / 2} \Gamma(z / 2) \zeta(z) \frac{\eta^{1 / 2-z} d z}{s-z}
$$

${ }^{3}$ We should take $\eta$ with $\arg \eta=\pi / 2-1 / \operatorname{Im} s$. 
with sufficiently large $\epsilon$. Omitting the details, we only mention that $\epsilon$ must exceed $\operatorname{Re} s$ and 1. Identity (4.1) is related to the functional equation (1.1) in a similar way.

There is yet another elegant method for the deduction of functional equations with free parameters, applicable in the cases where the zeta or $L$-function in question is related to some automorphic function by the Mellin transformation. For example, for $\zeta_{3}$ we have the representation

$$
\pi^{-s} \Gamma(s) \zeta_{3}(s)=\int_{0}^{\infty}(\vartheta(u)-1) u^{s-1} d u
$$

where $\operatorname{Re} s>3 / 2$, and the function

$$
\vartheta(u)=1+\sum_{n=1}^{\infty} r_{3}(n) \exp (-\pi n u)
$$

is analytic in the half-plane $\operatorname{Re} u>0$ and satisfies

$$
\vartheta(u)=u^{-2 / 3} \vartheta(1 / u) .
$$

Now, let a point $\eta \in \mathbb{C}$ with $\operatorname{Re} \eta>0$ be given. We replace integration along the real axis from 0 to $\infty$ in (4.4) with integration over a segment of the real line from 0 to some point $\xi>0$, then over some curve from $\xi$ to $\eta$, then from $\eta$ to some real point $\xi^{\prime}>\xi$, and then from $\xi^{\prime}$ to $\infty$ along the real axis (all paths must stay in the half-plane $\operatorname{Re} u>0$ ). This yields the representation

$$
\pi^{-s} \Gamma(s) \zeta_{3}(s)=\int_{\eta}^{\infty}(\vartheta(u)-1) u^{s-1} d u+\int_{0}^{\eta}(\vartheta(u)-1) u^{s-1} d u .
$$

In the first integral on the right in (4.7), we write $\vartheta(u)$ as in (4.5) and integrate termwise. In the second integral, we first write $\vartheta(u)$ as on the right-hand side of (4.6) and make the change $u \mapsto 1 / u$, and then integrate termwise. As a result, we extend $\zeta_{3}$ analytically to the entire complex plane, and we get the representation (4.1).

The general result of [15] can be stated as follows. Consider the following pair of functions defined by Dirichlet series

$$
L(a, s)=\sum_{n} \frac{a_{n}}{n^{s}}, \quad L(b, s)=\sum_{n} \frac{b_{n}}{n^{s}},
$$

which converge absolutely in the half-plane $\operatorname{Re} s>1$. Each of the sums in (4.8) is over some countable set of positive reals $n$, and all coefficient belong to $\mathbb{C}$. Of course, the functions $L(a, \cdot)$ and $L(b, \cdot)$ are holomorphic in the half-plane $\operatorname{Re} s>1$. We suppose that $L(a, \cdot)$ and $L(b, \cdot)$ extend to the entire complex plane up to meromorphic functions with at most finitely many poles. And finally, assume that the functions $L(a, \cdot)$ and $L(b, \cdot)$ are related to each other by an equation of Riemann type, namely, by the equation

$$
\Omega(s) L(a, s)=\Omega(1-s) L(b, 1-s), \quad s \in \mathbb{C},
$$

in which $\Omega$ is a product of several "gamma factors":

$$
\Omega(s)=\prod_{j=1, \ldots, k} \Gamma\left(\alpha_{j} s+\beta_{j}\right), \quad s \in \mathbb{C},
$$

with some $\alpha_{j}>0, \alpha_{j} \in \mathbb{R}$, and with arbitrary $\beta_{j} \in \mathbb{C}$. Put

$$
\alpha=\alpha_{1}+\cdots+\alpha_{k} .
$$

For $s \in \mathbb{C}$ and $u \in \mathbb{C}$ with $\operatorname{Re} u>0$, we denote

$$
E(s, u)=\frac{1}{2 \pi i} \int_{\sigma-i \infty}^{\sigma+i \infty} \Omega(s+z) u^{-\alpha z} \frac{d z}{z} .
$$


Here integration is done along the line from $\sigma-i \infty$ and $\sigma+i \infty$, where $\sigma$ is so large that the integrand has no singularities in the half-plane $\operatorname{Re} z \geq \sigma$. The condition $\operatorname{Re} u>0$ implies the absolute convergence of the integral in (4.12), which can be checked easily with the help of the Stirling formula. Also, the function $E$ is easily seen to be holomorphic in all its domain.

In accordance with Lavrik [15, we have

$$
\Omega(s) L(a, s)=\nabla_{a}(\eta, s)+\sum_{n} \frac{a_{n}}{n^{s}} E(s, \widetilde{n} \eta)+\sum_{n} \frac{b_{n}}{n^{1-s}} E\left(1-s, \widetilde{n} \eta^{-1}\right)
$$

for all $s \in \mathbb{C} \backslash P S$ and all $\eta \in \mathbb{C}$ with $\operatorname{Re} \eta>0$. Here, summation is over the same $n$ as in (4.8), $\widetilde{n}=n^{1 / \alpha}$ with $\alpha$ as in (4.11), and $P S$ is the set of all poles of the function $z \mapsto \Omega(z) L(a, z)$. The series on the right in (4.13) converge absolutely and uniformly on compact sets with respect to $s$, and the same is true for $\eta$. The parameter $\eta$ is free in the sense that the sum on the right-hand side of (4.13) is independent of $\eta$, despite the fact that each individual term does depend on $\eta$. By $\nabla_{a}(\eta, s)$ we mean the sum of the residues of the function

$$
z \mapsto \frac{\eta^{\alpha(s-z)}}{s-z} \Omega(z) L(a, z)
$$

at all points $p \in P S$. The function $\nabla_{b}$ defined similarly is related to $\nabla_{a}$ by the formula $\nabla_{b}\left(\eta^{-1}, 1-s\right)=\nabla_{a}(\eta, s)$. This follows from the functional equation (4.9) and "restores symmetry" on the right-hand side of (4.13).

Suppose that $u \in \mathbb{R}, u>0, s \in \mathbb{C}$, and $\operatorname{Re}\left(\alpha_{j} s+\beta_{j}\right)>0$ for all $j=1, \ldots, k$. Under these conditions, it was shown in [17] that the function $E$ can be written as

$$
E(s, u)=\int \ldots \int \exp \left(-t_{1} \cdots-t_{k}\right) t_{1}^{\alpha_{1} s+\beta_{1}} \ldots t_{k}^{\alpha_{k} s+\beta_{k}} \frac{d t_{1} \ldots d t_{k}}{t_{1} \ldots t_{k}},
$$

where integration is over the set of all points $\left(t_{1}, \ldots, t_{k}\right) \in \mathbb{R}^{k}$ with $t_{1}^{\alpha_{1}} t_{2}^{\alpha_{2}} \ldots t_{k}^{\alpha_{k}}>u^{\alpha}$, $t_{1}, \ldots t_{k}>0$. We see that $E$ can be treated as an "incomplete gamma function of multiplicity $k "$.

\section{$\S 5$. On methods of CAlCUlation}

The functional equations (4.13) with free parameters can be used for the calculation of values of $L$-functions. However, this is far from being simple. The problem is that, to use formula (3.14) for calculating values of an $L$-function, we need to have a fairly efficient procedure for calculating the values of $E$. For this function, we have the integral representations (4.12), (4.14), but they can hardly be called convenient from the viewpoint of calculations. Even if $k=1$, when, in essence, $E$ is the incomplete gamma function, the matter does not become simple. To calculate $\zeta(s)$ with $\operatorname{Im} s \sim T$ by formula (4.2), we need to compute $\gg \sqrt{T}$ terms of the series on the right-hand side, and in each of them we must calculate a value of the incomplete gamma function.

In some special cases, other methods of calculation are known, which employ certain specific properties of $L$-functions. For example, the Selberg-Chowla formula can be used. The values of the Riemann zeta function and, more generally, the Hurwitz zeta function can be calculated by using the Euler-Maclourin formula. In [19, 20], Odlyzko gained an impressive success in calculations related to the Riemann zeta function. To the best of our knowledge, Strömbergsson was the first to use functional equations with a free parameter for calculating the values (and zeros) of $L$-functions. Strömbergsson studied $L$-functions associated with the Maas wave forms, see 21. For these $L$-functions we have $k=2$, i.e., two gamma factors (see (4.9) and (4.10)) are present in the corresponding functional equation. A detailed analysis of problems and methods was given in [22] by Rubinstein. 
We turn to the function $\zeta_{3}$. To calculate its values by formula (4.1), we need a table for the coefficients $r_{3}(m), m=1,2, \ldots, R$. The required number $R$ of coefficients grows proportionally to the growth of $|\operatorname{Im} s|$. This makes no problem. We should choose the parameter $\eta$. We may take $\eta=\exp i(\pi / 2-\nu / T)$ for all $s$ with $T-T / 10 \leq|\operatorname{Im} s| \leq$ $T+T / 10$. Then $\eta$ becomes locally constant as a function of $s$, which is quite convenient. We may put $\nu=1$, or enlarge $\nu$, say, up to $\nu=10$. Increasing $\nu$, we reduce the required number $R$ of summands, but, simultaneously, raise the requirements concerning the precision of calculations. Difficulties only arise in connection with the incomplete gamma function.

Consider the sum

$$
\sum_{m=1}^{\infty} \frac{r_{3}(m)}{m^{s}} \frac{\Gamma(s, \pi m \eta)}{\Gamma(s)}
$$

occurring in (4.1). To calculate the values of the incomplete gamma function, we can use the identity

$$
\frac{\Gamma(s, z)}{\Gamma(s)}=1-e^{-z} \frac{z^{s}}{\Gamma(s)} \sum_{n=0}^{\infty} \frac{z^{n}}{\lambda_{n}(s)},
$$

where $\lambda_{n}(s)=(s+n) \lambda_{n-1}(s)$ for $n \geq 1$, and $\lambda_{0}(s)=s$. The calculations based on this formula are rather efficient under the condition $|z / s|<c$ with some constant $c<1$. If $|z / s|>c^{\prime}$ with some constant $c^{\prime}>1$, then to calculate $\Gamma(s, z)$ we can use the asymptotic expansion

$$
\frac{\Gamma(s, z)}{\Gamma(s)}=e^{-z} \frac{z^{s}}{\Gamma(s)} \sum_{n=1}^{\infty} \frac{\lambda_{n}(s)}{z^{n}},
$$

where $\lambda_{1}(s)=1$ and $\lambda_{n}(s)=(s-1) \ldots(s-n+1)$ for $n \geq 2$. Formulas (5.2) and (5.3) can be found in [23]. The calculation of $\Gamma(s, z)$ is more involved in the case where $|z / s|$ is close to 1 . Then the use of uniform asymptotic expansions is extremely undesirable. We employed the Nelson formula

$$
\Gamma(s, z+d)=\Gamma(s, z)-e^{-z} z^{s-1} \Delta(s, z, d),
$$

where $\Delta$ is a function that can easily be calculated; a precise description of it can be found in 24]. To make this clear, put $d=\pi \eta$. The Nelson formula allows us to calculate $\Gamma(s, \pi(m+1) \eta)$ once $\Gamma(s, \pi m \eta)$ is already calculated, say, with the help of (5.2). Next, we can repeat using formula (5.4) many times, till we calculate $\Gamma\left(s, \pi m^{\prime} \eta\right)$ with $m^{\prime}$ so large that the subsequent summands can be calculated by formula (5.3).

Once we know how to calculate the values of the function $\zeta_{3}$, the values of its derivative $\zeta_{3}^{\prime}$ and the zeros of both functions can be calculated by various methods, this makes no problem. We may use, e.g., the integral Cauchy formulas, or polynomial approximations.

\section{REFERENCES}

[1] N. V. Proskurin, On the cubic L-function, Algebra i Analiz 24 (2012), no. 2, 230-256; English transl., St.Petersburg Math. J. 24 (2013), no. 2, 353-370. MR3013333

[2] On the zeros of the zeta function of the Leech lattice, Zap. Nauchn. Sem. S.-Peterburg. Otdel. Mat. Inst. Steklov. (POMI) 404 (2012), 214-221; English transl., J. Math. Sci. (N.Y.) 193 (2013), no. 1, 124-128. MR3029602

[3] J.-P. Serre, Cours d'arithmétique, Pres. Univ. France, Paris, 1970. MR0255476 (41:138)

[4] E. Hecke, Über Modulfunktionen und Dirichletschen Reihen mit Eulerscher Productentwicklung. I, II, Math. Ann. 114 (1937), 1-28, 316-351. MR.1513142 MR.1513122

[5] G. Shimura, On modular forms of half-integral weight, Ann. of Math.(2) 97 (1973), $440-481$. $\operatorname{MR} 0332663(48: 10989)$ 
[6] C. L. Siegel, Contributions to the theory of the Dirichlet L-series and the Epstein zeta-functions, Ann. of Math.(2) 44 (1943), no. 2, 143-172. MR.0007760 (4:189c)

[7] K. Ramachandra and A. Sankaranarayanan, Hardy's theorem for zeta-functions of quadratic forms, Proc. Indian Acad. Sci. 106 (1996), no. 3, 217-226. MR1420165 (97j:11018)

[8] E. Bombieri and D. Hejhal, On the zeros of Epstein zeta functions, C. R. Acad. Sci. Paris Ser. I Math. 304 (1987), no. 9, 213-217. MR883477 (89c:11136)

[9] D. Hejhal, Zeros of Epstein zeta functions and supercomputers, Proc. Intern. Congress of Math., Vol. 1, 2 (Berkeley, Calif., 1986), Amer. Math. Soc., Providence, RI, 1987, pp. 1362-1384. MR934341 (89g:11128)

[10] E. C. Titchmarsh, The theory of functions, Oxford Univ. Press, 1939. MR3155290

[11] W. Müller, The mean square of Dirichlet series associated with automorphic forms, Monatsh. Math. 113 (1992), no. 2, 121-159. MR.1154475 (93g:11050)

[12] O. M. Fomenko, On Epstein's zeta-function. I, Zap. Nauchn. Sem. S.-Peterburg Otdel. Mat. Inst. Steklov. (POMI) 286 (2002), 169-178; English transl., J. Math. Sci. (N.Y.) 122 (2004), no. 6, 3679-3684. MR1937376 (2003j:11041)

[13] _ On Epstein's zeta-function. II, Zap. Nauchn. Sem. S.-Peterburg Otdel. Mat. Inst. Steklov. (POMI) 371 (2009), 157-170; English transl., J. Math. Sci. (N.Y.) 166, no. 2, 214-221.

[14] N. V. Proskurin, On the zeros of the zeta functions of a ternary quadratic form, Zap. Nauchn. Sem. S.-Peterburg Otdel. Mat. Inst. Steklov. (POMI) 392 (2011), 159-162; English transl., J. Math. Sci. (N.Y.) 184 (2012), no. 6, 753-754. MR2870224(2012m:11052)

[15] A. F. Lavrik, Functional equations of the Dirichlet functions, Izv. Akad. Nauk SSSR Ser. Mat. 31 (1967), no. 2, 431-442; English transl., Math. USSR-Izv. 1 (1967), no. 2, 421-432. MR0213310 $(35: 4174)$

[16] Approximate functional equations of Dirichlet functions, Izv. Akad. Nauk SSSR Ser. Mat. 32 (1968), no. 1, 134-185; English transl., Math. USSR-Izv. 2 (1968), no. 1, 129-179. MR.0223313 $(36: 6361)$

[17] N. V. Proskurin, On the problem of computing the values of L-functions, Zap. Nauchn. Sem. S.Peterburg. Otdel. Mat. Inst. Steklov. (POMI) 373 (2009), 273-279; English transl., J. Math. Sci. (N.Y.) 168 (2010), no. 3, 464-467. MR2749266 (2012b:11149)

[18] T. Chowla and A. Selberg, On Epstein's zeta-function, J. Reine Angew. Math. 227 (1967), 86-110. MR0215797 (35:6632)

[19] A. Odlyzko, New analytic algorithms in number theory, Proc. Intern. Congress of Math., vol. 1, 2 (Berkeley, Calif., 1986), Amer. Math. Soc., Providence, RI, 1987, pp. 466-475. MR934246 (89d:11117)

[20] , The $10^{22}$-nd zero of the Riemann zeta function, Dynamical, Spectral, and Arithmetic Zeta Functions, Contemp. Math., vol. 290, Amer. Math. Soc., Providence, RI, 2001, 139-144. MR.1868473 (2003h:11109)

[21] A. Strömbergsson, Studies in the analytic and spectral theory of automorphic forms, Uppsala Diss. Math., No. 17, Uppsala Univ., 2001. MR2715406

[22] M. Rubinstein M., Computational methods and experiments in analytic number theory, Recent Perspectives in Random Matrix Theory and Number Theory, London Math. Soc. Lecture Note Ser., vol. 322, Cambridge Univ. Press, Cambridge, 2005, pp. 425-506. MR2166470(2006d:11153)

[23] F. W. J. Olver, Asymptotics and special functions. Computer Science and Applied Mathematics, Acad. Press, New York, 1974. MR0435697 (55:8655)

[24] F. Oberhettinger and A. Erdelyi, Higher transcendental functions, vol. II, McGraw-Hill Book Company, Inc. 1953. MR0058756 (15:419i)

St. Petersburg Branch, Steklov Institute of Mathematics, Russian Academy of Sciences, Fontanka 27, St. Petersburg 191023, Russia

E-mail address: np@pdmi.ras.ru

Received 16/APR/2014

Translated by A. PLOTKIN 